\section{SAT0441 PREDICTIVE VALUE OF ANTIPHOSPHOLIPID ANTIBODIES IN THE ACUTE PHASE OF DEEP VEIN THROMBOSIS}

K. Perdan Pirkmajer ${ }^{1}$, A. Boc ${ }^{2}$, S. Čučnik ${ }^{1}$, A. Mavri ${ }^{3}$, P. Žigon ${ }^{1}$, E. Podovšovnik ${ }^{4}$, M. Štalc ${ }^{5}$, N. Vene ${ }^{3}$, A. Ambrožič ${ }^{1} .{ }^{1}$ Department of Rheumatology, University Medical Centre Ljubljana; ${ }^{2}$ Institute of Anatomy, Faculty of Medicine; University of Ljublj, ana; ${ }^{3}$ Department of Vascular Diseases, University Medical Centre Ljubljana, Ljubljana; ${ }^{4}$ Faculty for Tourism Studies University of Primorska, Capodistria; ${ }^{5}$ Department of Nuclear Medicine, University Medical Centre Ljubljana, Ljubljana, Slovenia

Background: Deep vein thrombosis (DVT) is frequent and potentially life threatening disease with tendency to reoccur. Anticoagulant treatment of the first episode of DVT usually lasts 3 months. Antiphospholipid syndrome (APS) is an important cause of DVT. However, the APS can be confirmed only 24 weeks after DVT according to the current APS classification criteria. ${ }^{1}$ Thus, undiagnosed APS patients, who cease anticoagulant therapy after 3 months, might be exposed to a greater risk for recurrent venous thromboembolism. Studies evaluating the significance of positive antiphospholipid antibody (aPL) test in the acute phase of DVT are lacking.

Objectives: To evaluate whether positive aPL test at the time of acute DVT diagnosis is predictive of APS.

Methods: Patients with acute DVT were included into a 24 month prospective study. All patients were given anticoagulants. aCL IgG/lgM and anti- $\beta 2 \mathrm{GPI} / \mathrm{lgG} /$ $\operatorname{lgM} / \lg A$ antibodies were determined by our in-house ELISA ${ }^{2}$ at inclusion and then every 4 weeks for the first 24 weeks. The last aPL measurement was performed 24 months after inclusion into the study. APS was confirmed if a patient tested positive (medium or high positive aCL and/or presence of anti- $\beta 2 \mathrm{GPI}) 12$ and 24 weeks after DVT. Lupus anticoagulants (LA) were tested after cessation of anticoagulation.

Results: 196 patients (111 male, 85 female, age $54 \pm 2$ years) included in the study had aPL titer assessed at least 5 times. Ultimately, 20/196 (10.2\%) patients fulfilled APS classification criteria. Among these, 15/20 (75\%) patients had medium or high titer aPL at the time of acute DVT ( 1 of them had double positive aPL and 2 of them had multiple positive aPL at first aPL determination). Two patients $(10 \%)$ had low positive aCL IgG and one had low titer aCL IgM. Two patients $(10 \%)$ were negative for aPL, but had later fulfilled APS criteria due to positive LA. APS was not established in 176/196 (89.8\%) patients. Among these, 146/176 (83\%) patients were negative for aPL at inclusion, while 30/176 (17\%) had low titer $\mathrm{aCL} \operatorname{lgM}$ or aCL IgG. Altogether, diagnostically important $\mathrm{aCL} \operatorname{lgG} /$ $\operatorname{lgM}$ and/or anti- $\beta 2 \mathrm{GPI}$ titer at the time of acute DVT had $83 \%$ specificity and $90.5 \%$ sensitivity for APS. Isolated low titer aCL IgG were more frequent in patients with APS than in patients without APS $(\chi 2=125.6 ; p<0.001)$. Completely negative $\mathrm{aCL} \operatorname{lgG} / \mathrm{lgM}$ and anti- $\beta 2 \mathrm{GPI}$ in the acute phase of DVT had a negative predictive value of $98.6 \%$.

Conclusions: Here we show that in acute phase of DVT, positive medium or high

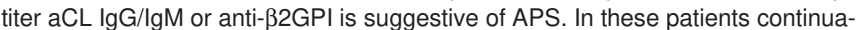
tion of anticoagulation beyond the initial 3 months should be considered. Patients with negative aPL in the acute phase of DVT do not need further aPL testing; however, LA should be determined. Low aPL titre at the time of acute DVT deems further testing imperative.

\section{REFERENCES:}

[1] Miyakis S, et al.Thromb Haemost 2006;4:295-306.2.

[2] Cucnik S, et al. Clin Chem Lab Med 2000;38:777-783.

Disclosure of Interest: None declared

DOI: 10.1136/annrheumdis-2018-eular.3912

\section{SAT0442 RISK FACTORS FOR HEART VALVE DISEASES IN PATIENTS WITH ANTIPHOSPHOLIPID SYNDROME}

K. Hiramoto, J. Kikuchi, Y. Kaneko, T. Takeuchi. Division of Rheumatology, Department of Internal Medicine, Keio University School of Medicine, Tokyo, Japan

Background: Antiphospholipid syndrome (APS) patients are known to be at a high risk of heart valve diseases (HVDs), however, risk factors of HVDs in APS patients is unclear.

Objectives: The purpose of this study is to elucidate risk factors for HVDs in patients with APS.

Methods: We reviewed consecutive APS patients diagnosed according to the Sydney criteria in 2006 who had been followed in Keio University Hospital in October 2017. The presence of HVDs was identified by the latest transthoracic and/or transesophageal echocardiography. HVDs were defined any kind of valve diseases such as mitral valve regurgitation (MR) and stenosis more than mild degree, aortic valve regurgitation (AR) and stenosis more than mild degree and Libman-Sacks endocarditis. Antiphospholipid antibodies (aPLs) included lupus anticoagulant (LA), anticardiolipin antibodies ( $\mathrm{aCL}$ ), and anticardiolipin $\beta 2$-glycoprotein I complex antibodies (aCL-32GPI). We divided the patients into two groups by the presence of HVDs, and evaluated the risk factors of them.

Results: Fifty-five APS patients were identified. Among them, 43 patients underwent echocardiography and enrolled in the analysis. Six patients were primary APS, 36 were secondary APS with systemic lupus erythematosus, and one with Sjögren syndrome. HVDs were detected in 26 (60.5\%) patients including 20 patients with MR, 8 patients with AR and a patient with Libman-Sacks endocarditis. The use of prednisolone, miscarriage episodes and the presence of arterial or venous thrombosis in patients with HVDs did not differ from those without HVDs Immunologically, there was no significant difference in anti-double-stranded DNA antibody, anti-Smith antibody, anti-ribonucleoprotein antibody, anti-SSA/La antibody and anti-SSB/Lo antibody between patients with or without HVDs. While the positivity of aCL or aCL- $\beta 2$ GPI was not different between patients with HVDs and those without, positive LA was much higher in patients with HVDs and in patients with MR than those without $(88.5 \%$ vs $35.3 \%, p<0.01 ; 85.0 \%$ vs $52.2 \%, p=0.027$ respectively). In addition, patients with HVDs had double and triple positive tests of aPLs significantly more frequently than those without HVDs $(p=0.010$ and $p=0.023$, respectively[Y1]). Hospitalisation risk in patients with HVDs because of heart failure or syncope were $0.011 /$ patient year.

\section{The proportions of positive aPLs in APS patients with HVDs and without them.}

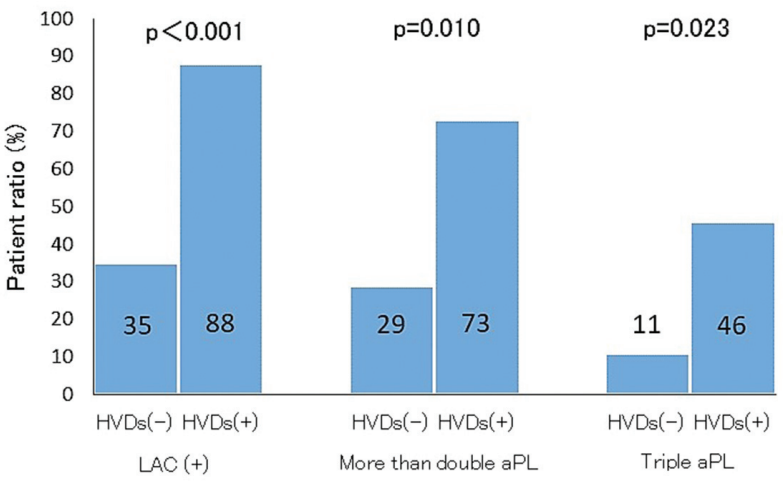

Conclusions: Positive LA test and positivity for 2 or more tests of aPLs are risk factors for HVDs in patients with APS. Regular monitoring cardiac ultrasonography is needed in those patients.

Disclosure of Interest: None declared

DOI: 10.1136/annrheumdis-2018-eular.5448

\section{SAT0443 DIAGNOSTIC AND PREDICTIVE EVALUATION USING SALIVARY GLAND ULTRASONOGRAPHY IN PRIMARY SJOGREN'S SYNDROME}

K.-A. Lee, S.-H. Lee, H.-R. Kim. Konkuk University Medical Center, Seoul, Korea, Republic Of

Background: There is an increasing need for alternative, non-invasive and reliable diagnostic tools with the potential to improve and simplify the diagnostic process for primary Sjogren's syndrome (pSS). The main advantage of salivary gland ultrasonography (SGUS) is the direct visualisation of structural abnormalities of the salivary glands. Despite these advantages of SGUS, a number of obstacles remain. Different SGUS scoring systems in B-mode were used in previous studies. The diagnostic usefulness of Doppler analysis and glandular size measurement has not been established. Indeed there is no proven prognostic factor for glandular damage in pSS, although a number of studies have revealed the risk factors for lymphoma.

Objectives: We aimed to assess the diagnostic value of SGUS as a single test for the detection of pSS in an integrated manner. We assessed the diagnostic accuracy of three SGUS parameters: the ultrasound (US) grey-scale scoring sys tem, glandular volume measurement, and intraglandular power Doppler US. The secondary aim was to examine the prognostic factors for severe structural changes in major salivary glands based on the SGUS scoring system.

Methods: Patients with pSS $(n=94)$ and idiopathic sicca syndrome $(n=44)$ were evaluated using the SGUS 0-48 scoring system, which comprises five parameters: parenchymal echogenicity, homogeneity, hypoechoic areas, hyperechogenic reflections, and clearness of posterior borders (figure 1). The salivary gland volume and intraglandular power Doppler signal (PDS) were also assessed. A multivariate linear regression analysis was performed to determine the factors associated with SGUS score. 
Results: Patients with pSS showed a significantly higher SGUS score than controls [median (IQR): 24.5 (13.0) vs 6 (3.75), $p<0.001$ ]. An SGUS cut-off of $\geq 14$ had a sensitivity of $80.9 \%$ and a specificity of $95.5 \%$ for the diagnosis of $p S S$. There were no significant differences in the measured volumes and PDS between pSS patients and controls. The SGUS score correlated with unstimulated salivary flow rate (USFR), serum rheumatoid factor and IgG. Double seropositivity with anti-Ro/ SS- $A$ and anti-La/SS-B $(\beta=6.060, p=0.001)$ and USFR $(\beta=-1.913, p<0.001)$ were independently associated with the SGUS score.

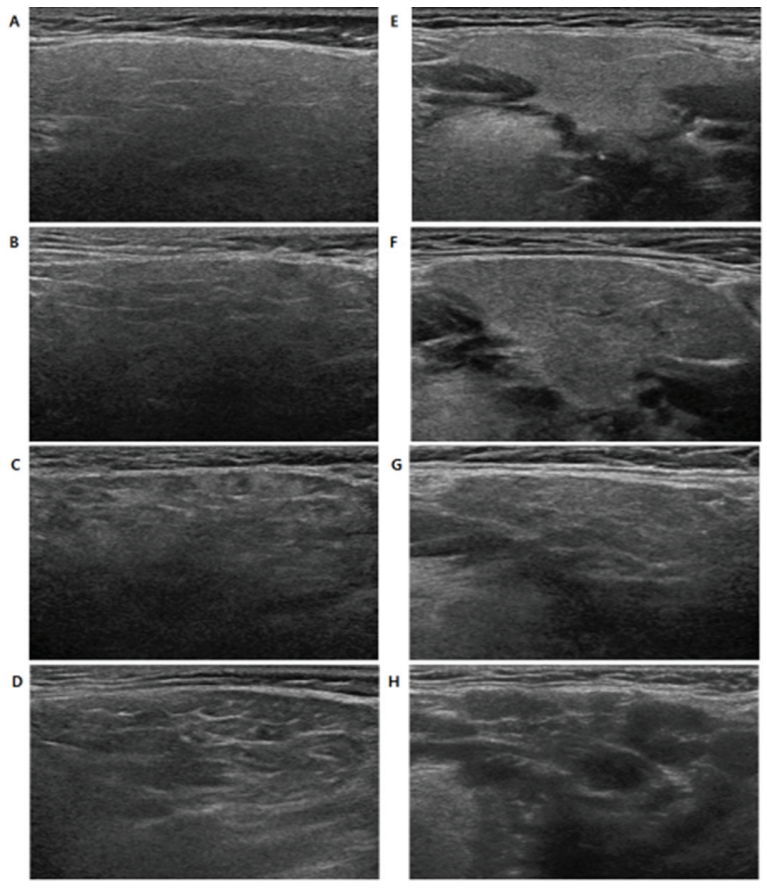

Conclusions: The SGUS scoring system is a valuable diagnostic method for pSS. Double seropositivity of anti-Ro/SS-A and La/SS-B is an independent predictive factor for structural damage of the salivary glands.

\section{REFERENCES:}

[1] Shiboski CH, Shiboski SC, Seror R, Criswell LA, Labetoulle M, Lietman TM, et al. 2016 American College of Rheumatology/European League Against Rheumatism classification criteria for primary Sjogren's syndrome: A consensus and data-driven methodology involving three international patient cohorts. Ann Rheum Dis 2017;76:9-16.

[2] Jousse-Joulin S, Milic V, Jonsson MV, Plagou A, Theander E, Luciano N, et al. Is salivary gland ultrasonography a useful tool in Sjogren's syndrome? A systematic review. Rheumatology (Oxford) 2016;55:789-800.

Acknowledgements: This paper was supported by Konkuk University in 2017 Disclosure of Interest: None declared

DOI: 10.1136/annrheumdis-2018-eular.2863

\section{SAT0444 INCIDENCE AND PREDICTORS OF IMMUNOSUPPRESSANT DISCONTINUATION AND RISK OF SUBSEQUENT FLARE IN PATIENTS WITH SYSTEMIC LUPUS ERYTHEMATOSUS}

M. Zen, F. Saccon, M. Gatto, M. Larosa, L. laccarino, A. Doria. Department of Medicine, Division of Rheumatology, University of Padova, Padova, Italy

Background: Prolonged treatment with immunosuppressants (IS) has been associated with long-term complications in Systemic Lupus Erythematosus (SLE); however, few data on IS discontinuation in remitted patients are available to date.

Objectives: We conducted an observational study to describe the proportion of SLE patients who discontinued IS and to assess the potential predictors of a subsequent flare.

Methods: We used data from Padua Cohort, which includes 454 SLE patients followed up from 1990 to 2017. Patients treated with IS over the disease course who discontinued IS and seen at least once in 2017 were studied. Reasons for discontinuation were: remission (defined by clinical SLE disease activity Index=0) or poor compliance/intolerance. Flares were defined according to SLEDAI Flare
Index. Predictors of a subsequent flare were analysed by multivariate logistic regression analysis.

Results: Eligible patients who were ever treated with IS were 297. IS were dis continued in 106 patients (35.7\%): mycophenolate (50, 47.2\%), azathioprine (27, $25.5 \%)$, cyclophosphamide $(11,10.4 \%)$, methotrexate $(10,9.4 \%)$, cyclosporine $(8,7.5 \%)$. Mean \pm SD follow-up duration after IS withdrawal was $82 \pm 64$ months (range 6-320).

83 out of 106 patients (78.4\%) discontinued IS due to remission (mean remission duration at IS discontinuation $39 \pm 28$ months), and $23(21.6 \%)$ due to poor compliance/intolerance. Among remitted patients, 18 (78.3\%) experienced a flare after IS discontinuation (9/55 patients with nephritis, 5/10 with arthritis, 2/9 with skin involvement, $1 / 3$ with neuroSLE, $1 / 4$ with haematological involvement) after a mean of $65 \pm 52$ months (range $6-180$ ). Conversely, in patients with poor compliance/intolerance, 17 relapsed (73.9\%) after a mean of $22 \pm 16$ months. Flare-free 10 year-survival rate was higher in patients who discontinued IS due to remission than to poor compliance/intolerance $(p<0.001$, figure 1$)$.

In patients who discontinued IS due to remission, a shorter duration of remission at IS discontinuation was associated with disease relapse $(p=0.006)$. Patients who were on IS due to nephritis had a lower risk of flare after IS discontinuation compared with patients with other manifestations $(16.4 \%$ vs $32.1 \%$, OR 0.58 $95 \% 0.32-0.98, p=0.049)$; patients with arthritis were those who were more likely to flare (OR 4.61, 95\% Cl 1.16-18.29, $\mathrm{p}=0.035$ ). Positive anti-SSA/SSB (OR $0.45,95 \% \mathrm{Cl} 0.26-0.78, \mathrm{p}=0.012$ ) and antimalarials intake after IS discontinuation (OR $0.22,95 \% \mathrm{Cl} 0.07-0.73, \mathrm{p}=0.015$ ) were associated with a lower risk of flare. No clinical features over the disease course were associated with flare occurrence.

At multivariate analysis, antimalarial use was the strongest protective factor against flares after IS discontinuation (OR $0.22,95 \% \mathrm{Cl} 0.05-0.85, \mathrm{p}=0.029$ ).

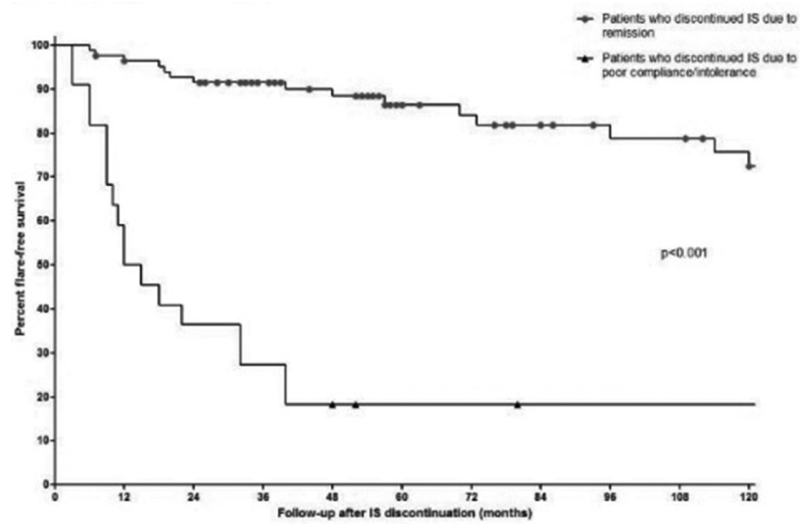

Abstract SAT0444 - Figure 1. Free-flare 10 year survival in the cohort.

Conclusions: In our cohort, one third of patients treated with IS discontinued the drug during the follow-up, in most cases due to a prolonged remission. Patients who discontinued IS due to remission had a higher free-survival rate than those who discontinued these drugs due to poor compliance/intolerance. The use of antimalarials after IS discontinuation was independently associated with a significant decrease in the risk of flare. IS discontinuation in patients with arthritis requires particular caution.

Disclosure of Interest: None declared

DOI: 10.1136/annrheumdis-2018-eular.6299

\section{SAT0445 THE CORRELATION BETWEEN THE TYPE OF CELLS IN MINOR SALIVARY GLANDS INFILTRATES AND THE SELECTED IMMUNOLOGICAL, CLINICAL AND LABORATORY PARAMETERS, IN PRIMARY SJOGREN'S SYNDROME PATIENTS WITH HISTORY OF EPSTEIN - BARR VIRUS INFECTION}

M. Maślińska ${ }^{1}$, M. Prochorec-Sobieszek ${ }^{2}$, B. Kwiatkowska ${ }^{1}$. ${ }^{1}$ Early Arthritis Clinic, National Institute of Geriatrics, Rheumatology and Rehabilitation; ${ }^{2}$ Department of Hematology Diagnostics, Institute of Hematology and Transfusion, Warsaw, Poland

Background: The number of inflammatory mononuclear cell foci is crucial in primary Sjogren's syndrome (pSS) diagnosis, with their cellular composition chang ing in subsequent inflammation stages. The Epstein -Barr virus (EBV) infection is believed to play a role in the pathogenesis of pSS. 